\title{
REPRESENTATION OF 3-D VOLUMETRIC OBJECT FROM THE PANTOMIME EFFECT AND SHADING CUES IN HUMAN BRAIN
}

\author{
QI ZHANG* and KEN MOGI ${ }^{\dagger}$ \\ Sony Computer Science Laboratories, Inc. \\ Takanawa Muse Bldg., 3-14-13, Higashigotanda \\ Shinagawa-ku, Tokyo 141-0022, Japan \\ *zhangq@csl.sony.co.jp \\ †kenmogi@csl.sony.co.jp
}

\begin{abstract}
Human ability to process visual information of outside world is yet far ahead of manmade systems in accuracy and speed. In particular, human beings can perceive 3-D object from various cues, such as binocular disparity and monocular shading cues. Understanding of the mechanism of human visual processing will lead to a breakthrough in creating artificial visual systems. Here, we study the human 3-D volumetric object perception that is induced by a visual phenomenon named as the pantomime effect and by the monocular shading cues. We measured human brain activities using fMRI when the subjects were observing the visual stimuli. A coordinated system of brain areas, including those in the prefrontal and parietal cortex, in addition to the occipital visual areas was found to be involved in the volumetric object perception.
\end{abstract}

Keywords: 3-D volumetric object; perception; pantomime effect; shading cues; fMRI.

\section{Introduction}

Human ability to process visual information of outside world is yet far ahead of man-made systems in accuracy and speed. Understanding of the mechanism of human visual processing will lead to a breakthrough in creating smarter artificial visual systems and accomplishing more efficient pattern recognition in the same way as human beings. Object perception is an important issue with long history in vision research. In particular, volumetric object perception that brings about the perception of thickness is one of the interesting topics in object perception. 3-D volumetric objects can be perceived via many types of inducing cues. The monocular cues such as shading, texture, are the natural ones to depict 3-D volumetric objects in paint works and photos, and are widely used in the computer graphics. On the other hand, binocular disparity is one of the important cues to perceive 3-D volumetric objects in the real world.

Several previous researches ${ }^{6,10,12,13}$ reported that the lateral occipital complex (LOC) area is involved in the object perception. In addition, researches on binocular 
stereopsis have reported that sensitivity to binocular disparity were found in many extrastriate areas, including V2, V3, V3A, VP, MT, MST and IT. ${ }^{1,19}$ Brouwer et al. reported that all visual areas responsive to disparity-defined depth. ${ }^{2}$ Neurons in $\mathrm{V} 4,{ }^{7}$ in middle temporal cortex ${ }^{14}$ and the caudal intraparietal sulcus ${ }^{18}$ are sensitive to the orientation of disparity-defined planes, and neurons in inferior temporal cortex (IT) are sensitive to the shape and curvature of disparity-defined surfaces. ${ }^{8}$ These fit with the idea that higher brain areas extract more complicated features from the patterns of retinal disparities, and integrate stereo information into different perceptual systems.

On the other hand, a phenomenon named as "shape from shading" is well known, where the monocular shading cues can induce 3-D shape perception, and this perception is reported as an "early" visual process computed in the occipital areas and is mostly a bottom-up mechanism. ${ }^{11,17}$

Furthermore, objects are usually perceived based on the direct physical cues. However, sometimes they are perceived in an alternative and indirect fashion, e.g. through the hands of pantomimists in pantomimes. Previously, one of the authors reported the pantomime effect in visual perception, ${ }^{21}$ where a 3 -D volumetric object was induced by suitably related and stereoscopically displayed objects. These objects were displayed with binocular disparities and were observed with binocular viewing. The most significant difference from the previous researches is that the perceived object is not only induced by the binocular disparities, but also induced by the interrelationships among the inducing objects. What is the brain mechanism for the 3-D object perception induced by the pantomime effect and the monocular shading cues? To pursue the answer, we conducted the fMRI experiments to measure the brain activities when the subjects were observing these two types of visual stimuli.

\section{Experimental Methods}

\subsection{Subjects and experimental protocols}

Five healthy right-handed volunteers (aged 23-30 years; male) with normal stereoscopic vision ability took part in the fMRI experiments. All subjects gave informed written consent for participation. The subjects were tested in advance to ensure that they could attain the 3 -D stereoscopic perception under the experimental conditions.

\subsection{Stimuli}

Stimuli from the pantomime effect are used in our experiments. In the pantomime effect, a 3-D volumetric object is perceived from some stereoscopically displayed inducing objects based on both binocular disparity and spatially distributed relations. The typical example of the stimuli in stereogram is shown in Fig. 1(a). The inducing objects are suitably related and arranged in 3-D space without occlusion 


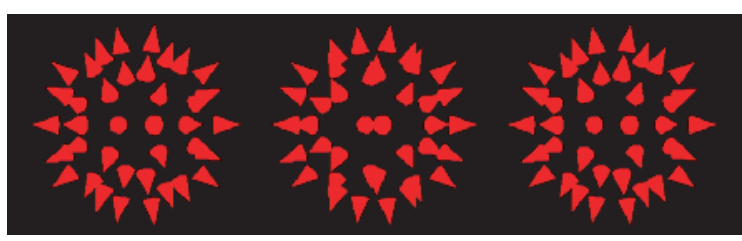

L $\mathrm{R}$

$\mathrm{L}$

(a)

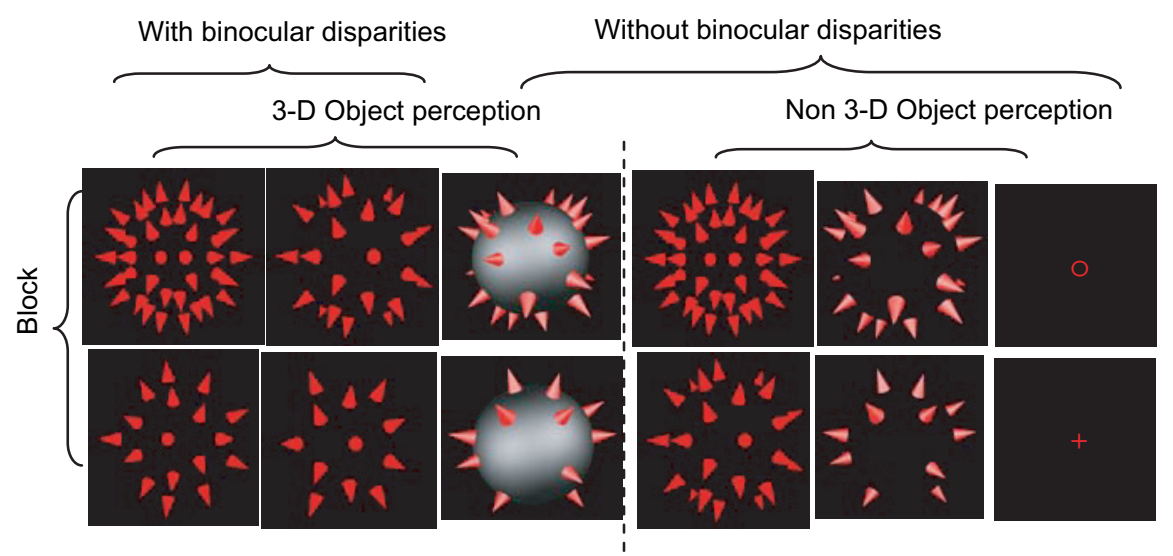

(b)

Fig. 1. (a) An example stereogram of the typical perception in the pantomime effect for an intact transparent sphere. (L: left eye image, R: right eye image; left and centre are for uncrossed fusion, while centre and right are for crossed fusion.) (b) The conceptual figure for the six types of stimuli used in the fMRI experiments. The first two columns of images were displayed with disparities for the two eyes, and subjects attained 3-D perception by observing through binocular glasses. The other columns were displayed without disparities, namely the image for the left eye was the same as that for the right eye, and the subjects obtain 2-D perception even when they observed through binocular glasses. In addition, for the left 3 columns of images, a 3-D object can be perceived, while no 3 -D object is perceived for the right 3 columns.

relationship, and they are perceived to sustain the 3-D illusory volumetric object in the same way as the hands of pantomimists. In this sense, this phenomenon is called the pantomime effect. The 3-D illusory object is perceived as a sphere with its inside brightness different from that of the surrounding space, as if it is "filled" with transparent medium and to occupy volumetric space, not simply a spherical surface. There is no direct physical stimulus on the perceived sphere, not like a shaded sphere with direct brightness stimulus on it. As the comparison, a 3-D volumetric sphere induced by monocular shading cues was adopted.

Figure 1 shows the six types of stimuli which were applied in our fMRI experiments. These stimuli include that for volume perception induced by both pantomime effect and monocular shading cues. The two left columns of images were displayed with disparities for the two eyes, and subjects stereoscopically attained 3 -D perception by observing through binocular glasses. The other columns were 
displayed without disparities, namely the image for the left eye was the same as that for the right eye. Subjects obtain 2-D perception even when they observed through binocular glasses. For detailed description, the first column represents the stimulus for the 3-D volumetric object. The upper one is for an intact transparent sphere, and the lower is a hemisphere. The second column is an opaque volumetric object and acted as a null stimulus in the experiments. The third column is the 3 -D object induced by monocular shading cues. For the upper image, more cones stand on the sphere, and the lower one with fewer cones. The fourth column acts as a control stimulus of 2-D perception with the similar retina projection. The images to both eyes are identical and the same as that for the left eye in the first column, thus only some triangles on a plane are perceived due to the absence of binocular disparity. The fifth column is the control stimulus where no 3-D sphere is perceived except for the same cones as in the third column, and it is also a null stimulus in the experiments. The last column is the fixation point stimulus. The upper is a circle, and the lower is a crosshair.

These stimuli were presented to the subjects using the Avotect Silent Vision SV-4021 fiber optic visual system, so that the subjects could easily attain the stereoscopic perception of the 3-D object induced by the binocular disparity. Subjects observed the visual stimuli through binocular glasses which were attached to the Avotec SV-4021 2-channel separated fiber optic binocular display system. The glasses were in SVGA resolution and fixed on a standard head coil of the fMRI system, above the subject's head when he/she was lying on his/her back. The stereo stimuli with binocular disparity were created using OpenGL in the situation where a sphere with the diameter of $110 \mathrm{~mm}$ was observed from $500 \mathrm{~mm}$ in distance, and the interpupillary distance was set as $60 \mathrm{~mm}$. The height of the cones was set as $21 \mathrm{~mm}$, and the diameter of the bases for cones was $12 \mathrm{~mm}$. In the experiments, the stimuli for the left eye and the right eye were displayed through independent 2-channel glasses. The interpupillary distance and the refractive correction (focus) for each piece of the glasses were adjusted for each subject to ensure the correct fusion for the stereoscopic perception and a clear observation. There was an eye relief (about $20 \mathrm{~mm}$ ) from the eye to the eyepiece, and it was a little different for each subject. In addition, for the stereo stimuli, the cones were unsymmetically standing on the surface of the sphere, pointing to a certain direction in 3-D space. Hence, it was difficult to know the precise visual angle of the stimuli, but an approximate value was $16^{\circ}$. The field of view of the binocular glasses was $30^{\circ}$ horizontal $\times 23^{\circ}$ vertical (nominal). The stimuli were displayed in red color of $[255,0,0]$ in RGB mode. For the shading stimuli, two white lighting sources were introduced for lighting a sphere of diffused materials.

\subsection{Experimental protocols}

The six types of stimuli shown in Fig. 1(b) were divided in six blocks and each block contained two types of stimuli for the same kind of perception. Each stimulus 
image was displayed in red color on a black background for two seconds in random order, followed by a $0.5 \mathrm{~s}$ interval, and repeated six times in one block. One block lasted $30 \mathrm{~s}$ with twelve images. One session consisted of all the six blocks repeating three times, and lasted nine minutes. The blocks also appeared in random order in one session.

The brain activities in which we are interested in this paper are those activated by the visual perceptions. Because the subjects were lying on their backs in a dark room, the pure observation task would make them feel sleepy. In order to prevent them from falling asleep, they were asked to do a simple task during their observation of the visual stimuli. Subjects clicked a right or left button when they were presented the stimulus image each time. They clicked the left button when the upper image was displayed, and clicked the right button when the lower one was displayed. The clicking fingers were the right index and middle finger. The button assignment was reminded again before each session began. Since the two images are quite different, the subjects did not need any effect to discriminate them. The motor activity by the button press task should disappear by the subtraction between two conditions during the fMRI data analyses.

\section{4. fMRI data acquisition and analysis}

EPI images were acquired with a Shimadzu-Marconi ECLIPSE 1.5T Power Drive 250 fMRI System, and the TR (repetition time) $=2.5 \mathrm{~s}$, TE (echo time) $=55 \mathrm{~ms}$. $\mathrm{T} 2 *$-weighted functional images with $5 \mathrm{~mm}$ thick slices were obtained in the resolution of $3 \mathrm{~mm} \times 3 \mathrm{~mm}$, and 23 slices were acquired in one scan. The EPI data were analyzed using the SPM5 software (Wellcome Department of Cognitive Neurology, University College London). ${ }^{3}$ Functional data were preprocessed by slice time adjustment, realignment, coregistration, segmentation, normalization and smoothing. For the realignment, the estimation quality is 0.9 ; the separation and smoothing parameters were 4 and 5; and 2nd Degree B-Spline was used for interpolation. In the coregistration step where the structural and functional data were coregistered, normalized mutual information was used for the objective function of estimation; the separation parameters were 4 and 2 , and the tolerances and histogram were 0.02 and 7, respectively. The coregistered T2 anatomical structural images were segmented into gray and white matter images and bias-field corrected structural images were obtained. The spatial normalization parameters obtained in the segmentation were used to normalize both the functional and anatomical structural images. Finally, a $6 \mathrm{~mm}$ full-width at half maximum (FWHM) of the isotropic Gaussian smoothing kernel was applied in smoothing. Fixed-effects models were used at the individual subject level of analysis, and random-effects models were used for group-level analyses. A canonical HRF with time and dispersion derivatives was adopted as the basis function in the individual level of analysis, and an autoregressive AR(1) model was used during classical parameter estimation to account for the serial correlations in fMRI time series due to aliased biorhythms and unmodelled neuronal activities. In addition, the estimated time series data of translations and 
rotations which were obtained in the preprocessing of realignment step were used as regressors when fitting GLMs, so that the movement effects were discounted when looking for brain activations. In the group level of analysis, an informed basis set, consisting of the canonical HRF and its two partial derivatives with respect to time (onset latency) and dispersion, was used. This used three observations per subject and data were analyzed using a "One-way ANOVA" with three levels. The $t$-contrast of the positive loadings on the canonical HRF were considered significant at $p<0.003$.

\section{Experimental Results}

\subsection{3-D volumetric object from the pantomime effect vs 2-D perception}

In this paper, our interests are in the brain mechanism for 3-D perception. We first examine the cortical areas for the 3 -D volume perception induced by the pantomime effect, and compared the activities when the subjects observed the stimuli for the volume perception induced by the pantomime effect (Fig. 1(b), 1st column) to that for 2-D perception (Fig. 1(b), 4th column). Figure 2 shows the results of the grouplevel analysis using the random-effects models. We can see that besides the activities in the occipital visual areas, the left parietal and prefrontal cortex were activated. The significant activated cerebral areas are listed in Table 1. The main activated areas were located significantly in bilateral occipital visual areas (Brodmann Areas (BA) 17 and 18). The anatomical locations were lingual gyrus, middle occipital gyrus, and cuneus. The areas in the prefrontal cortex were located in the left BA 9 and 10 with the anatomical locations as the left medial and middle frontal gyrus, and the left putamen. The right anterior cingulate (BA 24, 32 and 42) also showed activations. In addition, the activated areas included the left angular gyrus and the left inferior parietal lobule (BA 39).

\subsection{Volumetric object from shading cues vs 2-D perception}

In contrast to the 3 -D perception induced by the pantomime effect, where the binocular disparity and the relationship among the inducing objects play important roles to the 3-D perception, the 3-D sphere perception induced by monocular shading cues results from the direct physical stimulus on the perceived sphere, such as the variation in brightness. Though either type of stimuli brings about the $3-\mathrm{D}$ perception, we wonder if their perceptual mechanisms in the brain are different. Therefore, we examine a similar stimulus [3rd column in Fig. 1(b)] where a sphere is perceived with several 3-D objects (cones in Fig. 1) standing on it. Both the sphere and cones are perceived as $3-\mathrm{D}$ objects induced by the monocular shading cues without any binocular disparity. Because our interests are the brain activity differences between the 3-D perception and 2-D perception, a 2-D control stimulus is necessary in the fMRI experiments. We had considered several choices, such as 


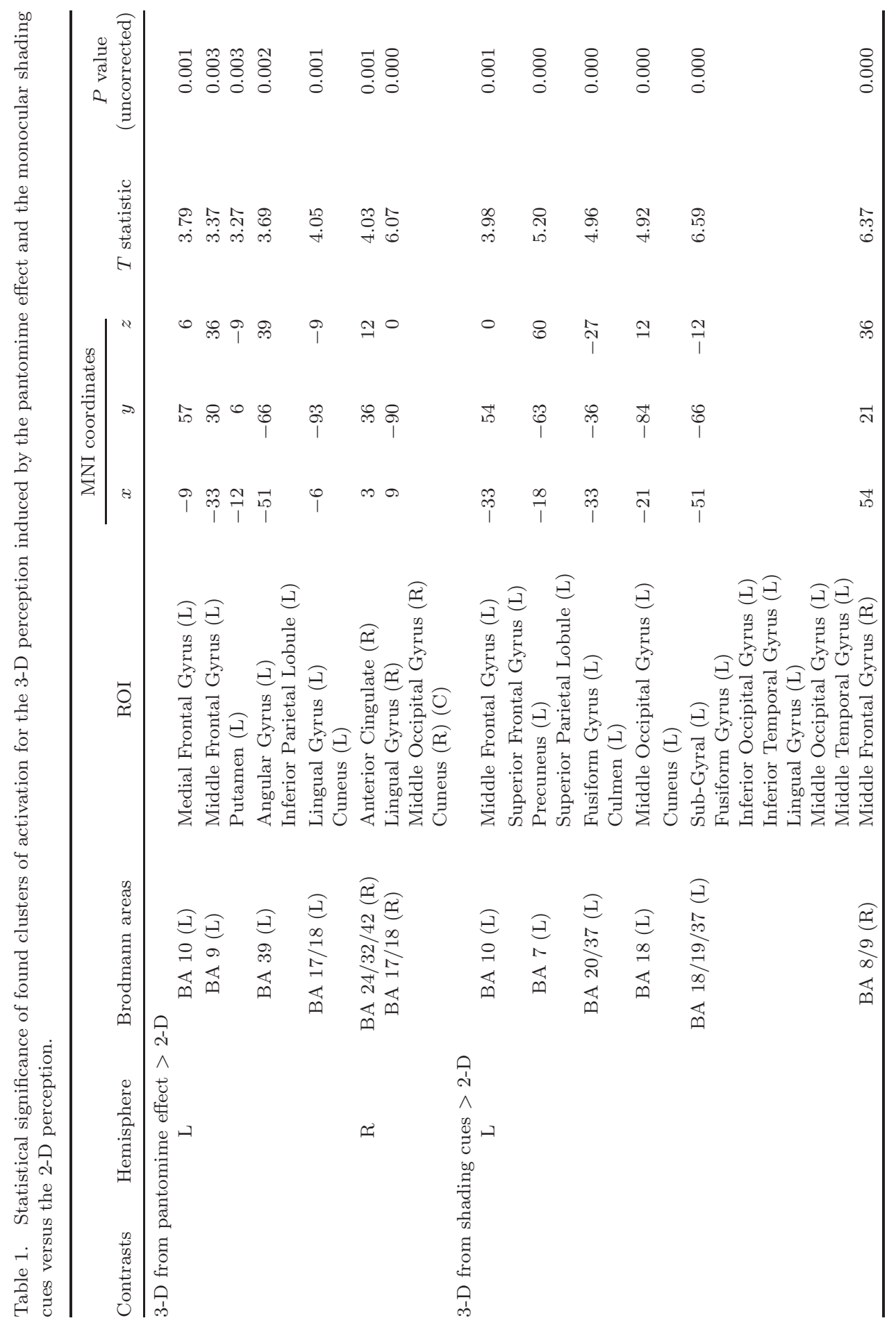




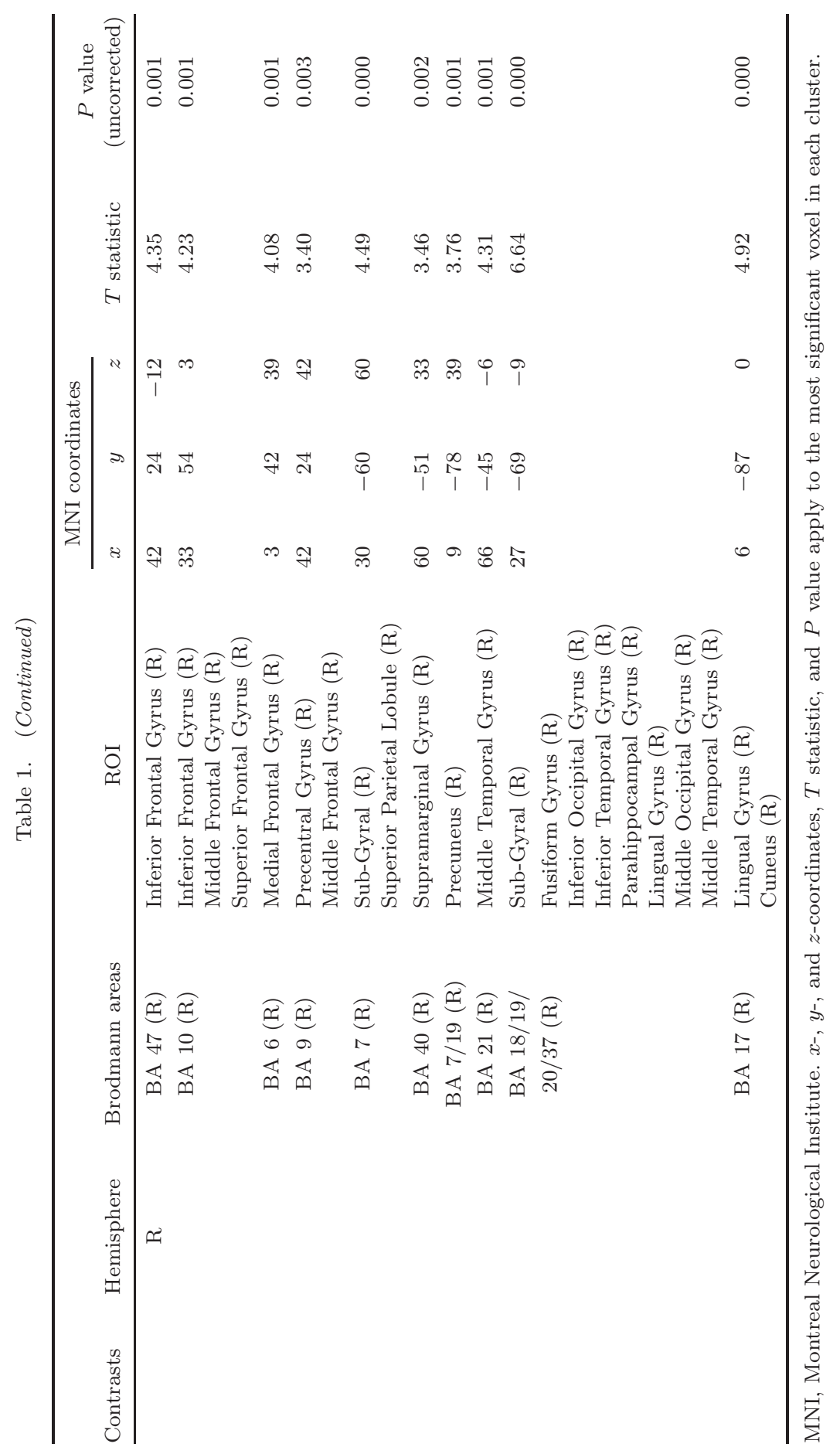




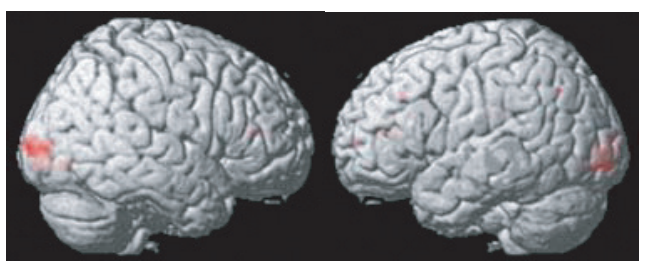

(a)
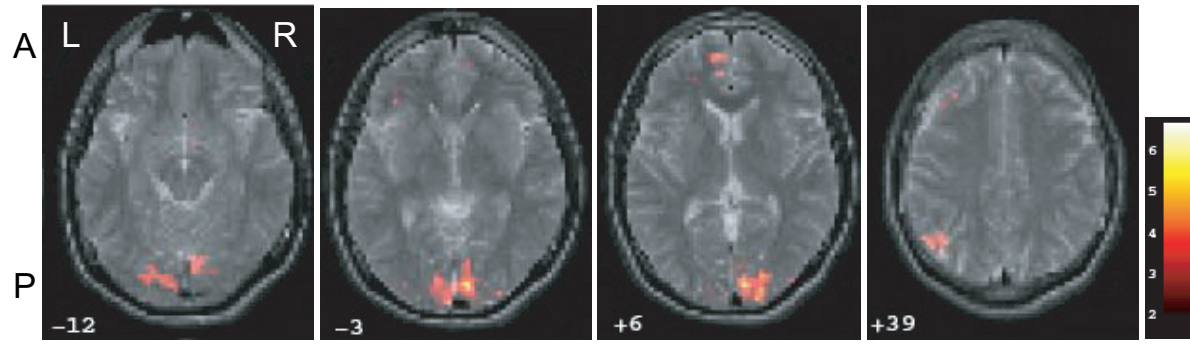

(b)

Fig. 2. fMRI experimental results of the group-level analysis using random-effects models. The color blobs represent the areas activated by the 3-D perception from the pantomime effect versus the 2-D perception. Besides the activities in the occipital visual areas, the left parietal and prefrontal cortex was activated. (a) Activities rendered on a 3-D brain $(t>3.33, p<0.003)$. (b) Activities plotted in four slices with statistic data $(t>2.0)$. The number in the figure shows the $z$ value of MNI coordinates. A: Anterior, P: Posterior, L: Left, R: Right.

a fixation point or some shaded cones but without a sphere. Since the physical differences in the stimuli between the 3-D perception and fixation point are too large, the differences in the brain activities could be considered due to the difference of the retina projection instead of the difference between the 3-D and 2-D perception. As for the stimulus of several shaded cones, because the shading cues on the cones even bring about 3-D perception for 3-D cones, it could not be used as the control of 2 -D perception. Finally, we used the same 2-D control as the previous section where neither binocular disparities nor shading cues are included [4th column in Fig. 1(b)]. We think that the difference in brightness between these two kinds of stimuli exactly express the different inducing ways for the 3-D perception and 2-D perception. The $3-\mathrm{D}$ perception is constructed through the processing of the brightness information.

The differences in the brain activities between these two kinds of stimuli are shown in Fig. 3. The activities were located in the bilateral occipital cortex including the lingual gyrus, inferior and middle occipital gyrus, cuneus, and sub-gyral (BA 17, 18, 19). Besides the occipital activation which were also observed in the pantomime effect perception, there were many activities in the occipito-temporal cortex, including the bilateral fusiform gyrus, inferior and middle temporal gyrus, and the right parahippocampal gyrus (BA 19, 20, 21, and 37). In addition, the 


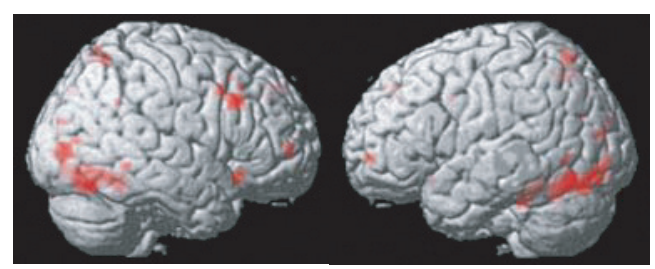

(a)
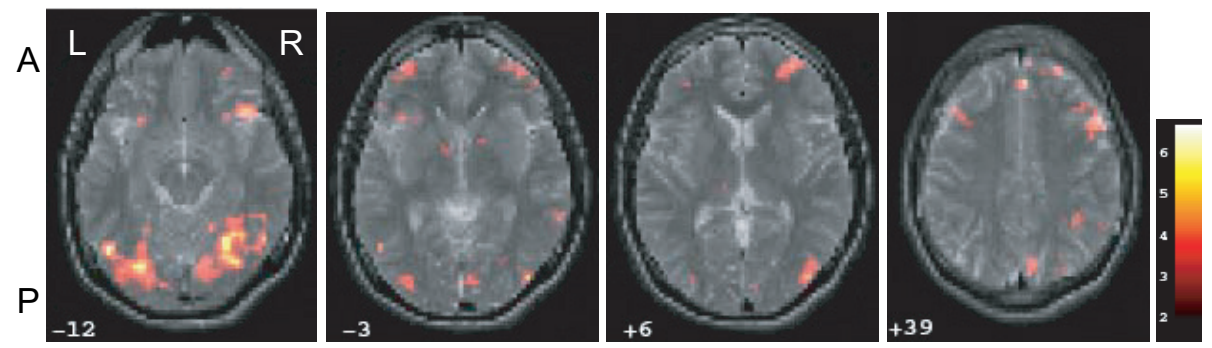

(b)

Fig. 3. fMRI experimental results of the group-level analysis using random-effects models. The color blobs represent the areas activated by the 3-D perception from the monocular shading cues versus the 2-D perception. Similar to the activation induced by the pantomime effect, there are activities in the prefrontal cortex. Particularly, the right prefrontal cortex is more activated. In addition, there are activations in the right parietal cortex. (a) Activities rendered on a 3-D brain $(t>3.33, p<0.003)$. (b) Activities plotted in four slices with statistic data $(t>2.0)$. The number in the figure shows the $z$ value of MNI coordinates. A: Anterior, P: Posterior, L: Left, R: Right.

parietal cortex was activated in the areas of bilateral precuneus and superior parietal lobule (BA 7), and the right supramarginal gyrus (BA 40). In the frontal cortex, there were also activities in the left middle and superior frontal gyrus (BA 10). In addition, there were more activities in the right frontal/prefrontal cortex, which were not observed in the condition of pantomime effect, including the inferior, middle, superior and medial frontal gyrus, and precentral gyrus (BA 6, 8, 9, 10 and 47). In summary, there are more right frontal and parietal activations, compared with the activities induced by the pantomime effect, where more left hemisphere activities were observed.

\subsection{Common areas and differences in the two types of volumetric object perception}

The above fMRI experimental results show that the frontal, parietal, as well as the occipital cortex were involved in both types of 3 -D volumetric object perception induced by the pantomime effect and the monocular shading cues. Both common and different cortical areas exist in the two kinds of 3-D perception. The common areas were located in the occipital visual cortex and the left prefrontal cortex. We propose that activities in the occipital cortex were in charge of the general visual 
processing to recognize the inducing objects, and the left prefrontal cortex in BA $9 / 10$ was involved in the consciousness awareness of the perception.

On the other hand, there are different brain areas for these two kinds of 3-D perception. Examples of group-level BOLD signal changes in these areas are shown in Fig. 4. For the perception induced by the pantomime effect, there were activities in the left parietal cortex [Fig. 4(a)] and the right anterior cingulate cortex (ACC) [Fig. 4(b)]. In contrast, the perception induced by the monocular shading cues activated more the right frontal/prefrontal and parietal cortex, as well bilateral occipito-temporal cortex. For the occipito-temporal cortex [Fig. 4(c)], the different areas were mainly located in the bilateral BA 19, 20, 37, which included bilateral fusiform gyrus, inferior and middle temporal gyrus, and the right parahippocampal gyrus. These areas were mainly in the ventral pathway of the visual processing system, and we propose that they were in charge of the processing for the brightness of the shading cues. As for the right parietal cortex [Fig. 4(d)], including the sub-gyral, right parietal lobule, precuneus, superior parietal lobule, and postcentral gyrus (BA 7, 40), we propose that these areas are in charge of the spatial processing for the 3-D perception. For the prefrontal cortex [Fig. 4(e)], the activities were located in the right prefrontal cortex of BA $6,8,9,10,47$. They were the right inferior, middle, superior and medial frontal gyrus, as well as the precentral gyrus. We suppose that these areas processed the information from the occipital and temporal cortex and constructed the 3 -D perception from the brightness information.

\subsection{Temporal features of volumetric object perception}

So far we have shown that different cortical areas are involved in these two types of 3-D perception, and supposed that their processing mechanisms were different. Since the different brain mechanisms may result in different processing time for attaining the perception, we studied the response time of the subjects when they attained the perception. The results are shown in Fig. 5, where the individual response time for the 3 -D perception induced by the pantomime effect and monocular shading cues, as well as the 2-D perception were plotted in different colors. The data for all five subjects were shown. For four of five subjects, the monocular shading cues took the longest time for the 3-D object perception, except for one subject (s2). The $t$-test results show that the response time did not have a significant difference for subject 2, whose response time for shading cues was shorter, while the differences for the other four subjects were significant. In addition, the response time for the 3-D perception induced by the pantomime effect was not significantly different from that for the 2-D perception, while the time induced by the monocular shading cues was significantly different from that for the 2-D perception. Considering that the perception from shading cues involves the higher-level brain cortical areas, we therefore propose that the perception of volumetric object from shading is not a simple early process, ${ }^{11,17}$ and the right prefrontal cortex processes 


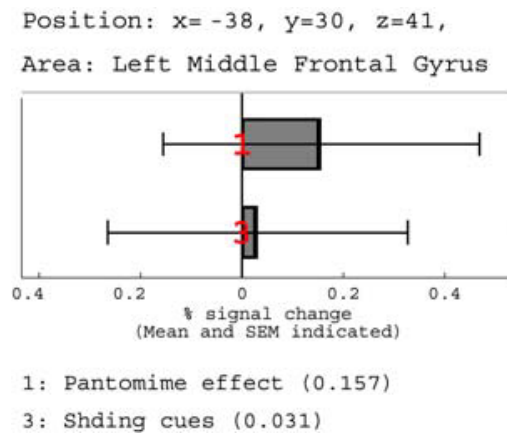

(a)

Position: $x=29, y=-64, z=-7$, Area: Right Fusiform Gyrus

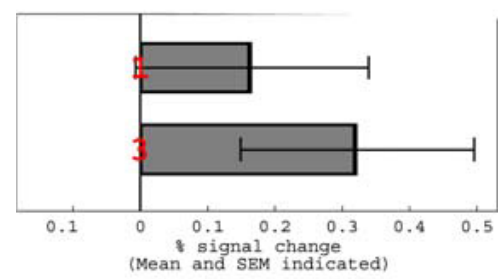

1: Pantomime effect $(0.166)$

3: Shading cues $(0.323)$

(c)
Position: $x=3, y=29, z=18$, Area: Right Anterior Cingulate Cortex

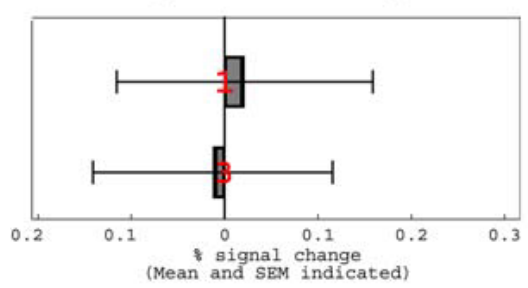

1: Pantomime effect $(0.022)$

3: Shading cues $(0.013)$

(b)

Position: $x=31, y=60, z=63$,

Area: Right Superior Parietal Lobule

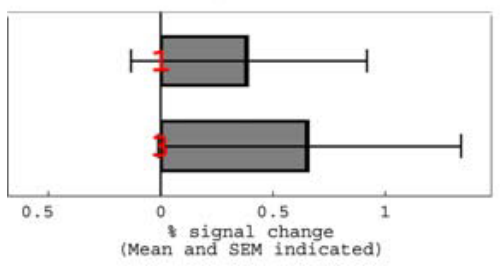

1: Pantomime effect (0.393)

3: Shading cues $(0.663)$

(d)

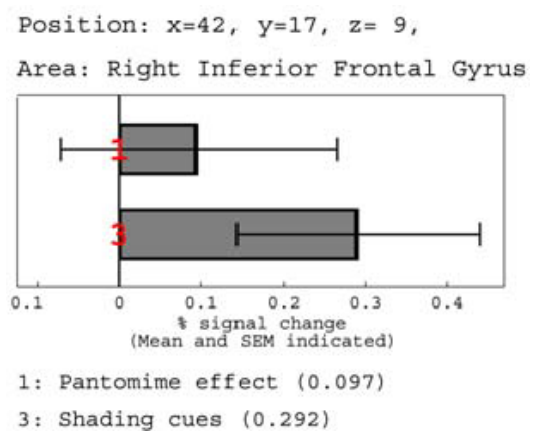

(e)

Fig. 4. The group-level BOLD signal changes corresponding to the 3-D perception induced by the pantomime effect and the monocular shading cues.

the information from the lower-level visual cortex, and projects a top-down signal back to construct a volumetric object perception. On the other hand, the perception from the pantomime effect takes almost the same time as the 2-D perception. It is accelerated by binocular information though it also recruits some higher-level cortex. 
Response time for 3D, shading, and 2D

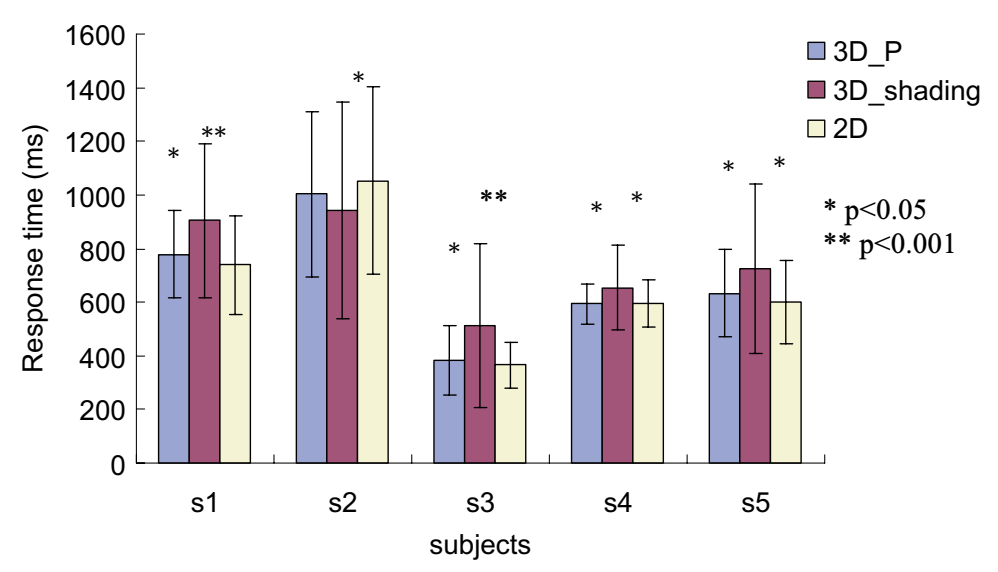

Fig. 5. Response times for different types of perception. The 3-D perception induced by the monocular shading cues takes the longest time.

\section{Discussions}

It is well known that visual system consists of two pathways: the ventral pathway is in charge of "what" information such as form, colour, texture, and so on; and the dorsal pathway deals with "where" or "how" issues, including the spatial and motion information. ${ }^{5,20}$ Several years ago, Goodale et al. studied an individual patient (DF) with visual form agnosia ${ }^{4}$, who was able to use vision to adjust the posture of her hand to the size, shape and orientation of a goal object as she reached out to pick it up - even though she showed no perceptual awareness of these same object attributes. Their fMRI experiments revealed no differential activation in ventral-stream areas which were severely damaged in DF when she was presented with line drawings of common or novel objects; but when DF reached out to grasp objects of different sizes and orientations, she showed relatively normal activation in dorsal-stream areas implicated in the visual control of grasping. They concluded that vision-for-perception and vision-for-action depended on quite separate visual pathways in the cerebral cortex. Here our fMRI results show that both the ventral and dorsal pathways were involved in 3-D volumetric object perception. We did not ask the subjects act on the 3-D perception. The activity for the button click was quite similar for every stimulus, and was thought to disappear by subtraction of two conditions. So the difference in the brain activities should be due to the visual observation. We provide the suggestion that maybe action probably does not need the ventral stream areas, but the visual perception indeed needs the dorsal stream areas, in particular, the parietal cortex for the spatial processing. The vision-forperception pathway should include both the ventral and partial dorsal pathways.

In addition, we found that there is laterality in the prefrontal cortex for the two perceptions. The perception induced by the pantomime effect specifically activated 
more the left hemisphere. In contrast, the monocular shading cues activated more the right prefrontal cortex. The right prefrontal cortex was reported in charge of the working memory processing during encoding process and retrieval process for nonverbally coded information ${ }^{16}$, and associated with recognition. ${ }^{15}$ Furthermore, Ramachandran pointed out that the processing of shape from shading depended on the previous experience of the light source illuminating. ${ }^{17}$ Hence the role of the right prefrontal cortex in the 3-D perception by shading cues is related with the working memory function. The information from lower-level cortex was encoded again here, together with the retrieval processing of previous experiences on the light illumination, to construct the 3-D object perception. Then top-down instructions were sent to the inferior temporal cortex. This is the reason why the perception induced by the shading cues takes longer time. Our findings extend the knowledge about the well-known "Shape from shading" perception, and show that object from shading also recruited higher-level processing in the parietal and prefrontal cortex, and include top-down processing.

On the other hand, Kourtzi et al. ${ }^{9}$ found that no adaption was observed between the observations of stereoscopically defined silhouettes and shaded 3-D images of the same objects, and suggested that the stereo and shaded object conditions may involve different neural populations in the LOC. Though our research did not study the details in LOC, we found that the processing for the stereo and shading cues activated quite different cortical areas, besides the occipital areas. So we think that the processing of the 3-D perception from different types of inducing cues might employ quite different mechanisms from the occipital LOC to the parietal and prefrontal cortex.

\section{Conclusions}

Our study revealed that the 3-D perception recruited a wide range of cortical areas, from the elementary processing in the occipital visual areas to parietal and prefrontal cortex, in particular for the 3-D perception induced by the monocular shading cues. Our fMRI experiments not only confirmed previous studies on the object perception in the occipital cortex, but also extended the knowledge of the 3-D processing in the prefrontal cortex, and clarified the mechanism for two different kinds of 3-D perception induced by the pantomime effect and monocular shading cues. For the perception induced by the pantomime effect, the left frontal/prefrontal cortex were more activated; in contrast, the right prefrontal/frontal cortex were more involved in the perception from shading. Furthermore, we compared their response times, and the perception induced by the shading cues took longer time than that by the pantomime effect. Hence we suppose that the shading cues need to be processed at the ventral pathway first and then forwarded to the right frontal cortex for extracting the spatial information to construct the 3-D perception. In contrast, the pantomime effect is related with the processing of stereopsis, and the perception is accelerated by binocular information. These findings suggest that we may 
need to handle the 3-D object pattern recognition in different algorithms for the stimuli induced by binocular and monocular cues when we create artificial visual systems.

\section{Acknowledgments}

The fMRI experiments were conducted at ATR, Kyoto, Japan. We thank Akiko Callan, Yasuhiro Shimada, Ichiro Fujimoto, Yuko Shakudo, Shinobu Masaki, and Kayoko Nakagawa at Brain Activity Imaging Center, for their support in acquiring the fMRI data, and helpful advice in experimental design and data analysis. We also thank the two reviewers for their insightful comments to improve this paper.

\section{References}

1. B. T. Backus, D. J. Fleet, A. J. Parker and D. J. Heeger, Human cortical activity correlates with stereoscopic depth perception, J Neurophysiol. 86 (2001) 2054-2068.

2. G. J. Brouwer, R. van Ee and J. Schwarzbach, Activation in visual cortex correlates with the awareness of stereoscopic depth, J Neurosci. 25 (2005) 10403-10420.

3. K. Friston, A. Holmes, K. Worsley, J. Poline, C. Frith and R. Frackowiak, Statistical parametric maps in functional imaging: a general linear approach, Human Brain Mapping 2 (1995) 189-210.

4. M. A. Goodale, T. W. James, J. C. Culham, G. K. Humphrey and A. D. Milner, fMRI studies of action and perception pathways in an individual with visual form agnosia, Perception 32 (Supplement) (2003) 15.

5. M. A. Goodale and A. D. Milner, Separate visual pathways for perception and action, Trends Neurosci. 15 (1992) 20-25.

6. K. Grill-Spector, T. Kushnir, S. Edelman, G. Avidan, Y. Itzchak and R. Malach, Differential processing of objects under various viewing conditions in the human lateral occipital complex, Neuron 24 (1999) 187-203.

7. D. A. Hinkle and C. E. Connor, Three-dimensional orientation tuning in macaque area V4, Nat. Neurosci. 5 (2002) 665-670.

8. P. Janssen, R. Vogels, Y. Liu and G. A. Orban, Macaque inferior temporal neurons are selective for three-dimensional boundaries and surfaces, J Neurosci. 21 (2001) 9419-9429.

9. Z. Kourtzi, M. Erb, W. Grodd and H. H. Bülthoff, Representation of the perceived 3-D object shape in the human lateral occipital complex, Cereb. Cortex 13 (2003) 911-920.

10. R. Malach, J. Reppas, R. Benson, K. Kwong, H. Jiang, W. Kennedy, P. Ledden, T. Brady, B. Rosen and R. Tootell, Object-related activity revealed by functional magnetic resonance imaging in human occipital cortex, PNAS 92 (1995) 8135-8139.

11. P. Mamassian, I. Jentzsch, B. A. Bacon and S. R. Schweinberger, Neural correlates of shape from shading, Neruoreport 14 (2003) 971-975.

12. J. D. Mendola, A. M. Dale, B. Fischl, A. K. Liu and R. B. H. Tootell, The representation of illusory and real contours in human cortical visual areas revealed by functional magnetic resonance imaging, J. Neurosci. 19 (1999) 8560-8572.

13. C. Moore and S. A. Engel, Neural response to perception of volume in the lateral occipital complex, Neuron 29 (2001) 277-286. 
14. J. D. Nguyenkim and G. C. DeAngelis, Disparity-based coding of three-dimensional surface orientation by macaque middle temporal neurons. J Neurosci. 23 (2003) 71177128.

15. S. F. Nolde, M. K. Johnson and C. L. Raye, The role of prefrontal cortex during tests of episodic memory, Trends Cogn. Sci. 2 (1998) 399-406.

16. B. Optiz, A. Mecklinger and A. D. Friederici, Functional asymmetry of human prefrontal cortex: encoding and retrieval of verbally and nonverbally coded information, Learning \& Memory 7 (2000) 85-96.

17. V. S. Ramachandran, Perception of shape from shading, Nature 331 (1988) 163-166.

18. M. Taira, K. I. Tsutsui, M. Jiang, K. Yara and H. Sakata, Parietal neurons represent surface orientation from the gradient of binocular disparity, J Neurophysiol. 83 (2000) 3140-3146.

19. D. Y. Tsao, W. Vanduffel, Y. Sasaki, D. Fize, T. A. Knutsen, J. B. Mandeville, L. L. Wald, A. M. Dale, B. R. Rosen, D. C. Van Essen, M. S. Livingstone, G. A. Orban and R. B. Tootell, Stereopsis activates V3A and caudal intraparietal areas in macaques and humans, Neuron 39 (2003) 555-568.

20. L. G. Ungerleider and M. Mishkin, Two cortical visual systems, in Analysis of Visual Behavior, eds. D. J. Ingle, M. A. Goodale and R. J. W. Mansfield (The MIT Press, Cambridge, MA, 1982), pp. 549-586.

21. Q. Zhang, M. Idesawa and Y. Sakaguchi, Pantomime effect in the perception of volumetric transparent illusory objects with binocular viewing, Jpn. J. Appl. Phys. 37 (1998) L329-L332.

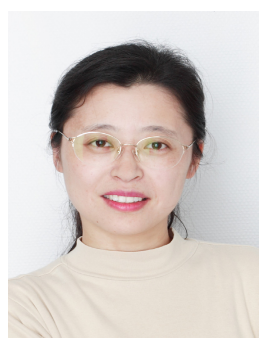

Qi Zhang received the B.E. and M.E. degrees in information science and electronic engineering from Zhejiang University, China, in 1990 and 1993 respectively, and the Ph.D. in information systems from The University of Electro-Communications, Japan, in 1998. Currently, she is working in the field of systems brain science with Sony Computer Science Laboratories, Japan.

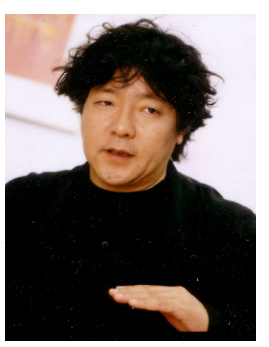

Ken Mogi received his Ph.D from Department of Physics, University of Tokyo. He conducted research in brain science in Riken (Japan) and the University of Cambridge (U.K.). Currently, he is a senior researcher at Sony Computer Science Laboratories, Tokyo, and is a visiting Professor at Tokyo Institute of Technology. 